\title{
Metachronous Colon Metastasis to the Thyroid: A Case Report and Literature Review
}

\author{
Dvir Froylich, ${ }^{1}$ Eitan Shiloni, ${ }^{1}$ and David Hazzan ${ }^{2}$ \\ ${ }^{1}$ General Surgery B Department, Carmel Medical Center, 34362 Haifa, Israel \\ ${ }^{2}$ Minimally Invasive Surgery, Surgery B Department, Carmel Medical Center, 34362 Haifa, Israel \\ Correspondence should be addressed to Dvir Froylich; dvirfr7@gmail.com
}

Received 8 August 2013; Accepted 9 September 2013

Academic Editors: D. J. Bentrem and Y. Rino

Copyright (C) 2013 Dvir Froylich et al. This is an open access article distributed under the Creative Commons Attribution License, which permits unrestricted use, distribution, and reproduction in any medium, provided the original work is properly cited.

The thyroid gland is a known site for metastatic tumors from various primary sites. Thyroid metastases are not an exceptional finding at autopsy, and they are encountered in $2 \%$ to $9 \%$ of the patients with malignant neoplasm. The most frequent tumors to metastasize are breast, lung, melanoma, and kidney carcinomas. Despite the fact that it is one of the largest vascular organs in the body, clinical and surgical cases have given an incidence of $3 \%$ of secondary malignances of this organ. Metastatic colon carcinoma to the thyroid gland has been reported, and it is not as rare as one might think. We present a very unique case of colon carcinoma metastasis to the right thyroid lobe and lung five years after colon resection, with reoccurrence two years later in the contralateral thyroid lobe. The literature regarding colon cancer metastasizing to the thyroid gland was reviewed with an attempt to disclose features of this presentation regarding patient's prognosis.

\section{Introduction}

Microscopic metastasis to the thyroid gland is not rare, having been found in $4 \%-9 \%$ of autopsy studies [1]. The most frequent tumors to metastasize are breast, lung, melanoma, and kidney carcinomas [2]. However, gastrointestinal and particularly colonic metastases to the thyroid are extremely rare and usually associated with lung and liver metastasis [3]. A literature search identified 34 reported cases of colon carcinoma metastasis to the thyroid.

We present a case of colon carcinoma metastasis to the right thyroid lobe and lung five years after colon resection, with recurrence two years later in the contralateral thyroid lobe. To the best of our knowledge, metachronous colon metastasis to the thyroid has never been reported previously. This unique case is presented and the published literature of colonic carcinoma metastasized to the thyroid is reviewed.

\section{Case Presentation}

A 65-year-old female with hypothyroidism had a laparoscopic left colectomy due to sigmoid carcinoma (T3, N1, M0) in March 2005. Adjuvant chemoradiotherapy was administrated postoperatively and included FOLFIRI (5-FU, leucovorin and irinotecan) as well as pelvic radiation due to positive colonic margins. A CT scan performed four months after the surgery showed an enlarged right thyroid lobe with a nodule. Consequently, a PET CT was performed and revealed a pathological uptake in the right thyroid lobe. Fine needle aspiration (FNA) demonstrated normal cytology.

In December 2009, the patient's carcinoembryonic antigen (CEA) rose and a CT of the chest and abdomen demonstrated possible metastatic lesions in the right lung and pelvis. FNA of the lung showed adenocarcinoma originating in the colon.

In March 2010, the patient underwent a right upper and middle lobectomy followed by chest radiotherapy. Three months later, an anterior resection of the rectum was performed because of anastomotic recurrence and pelvic metastasis positioning on the sigmoid mesenterium. Xeloda (capecitabine) was given as adjuvant chemotherapy postoperatively.

A PET CT was performed in November 2010 and again demonstrated a pathological uptake in the right thyroid lobe. 


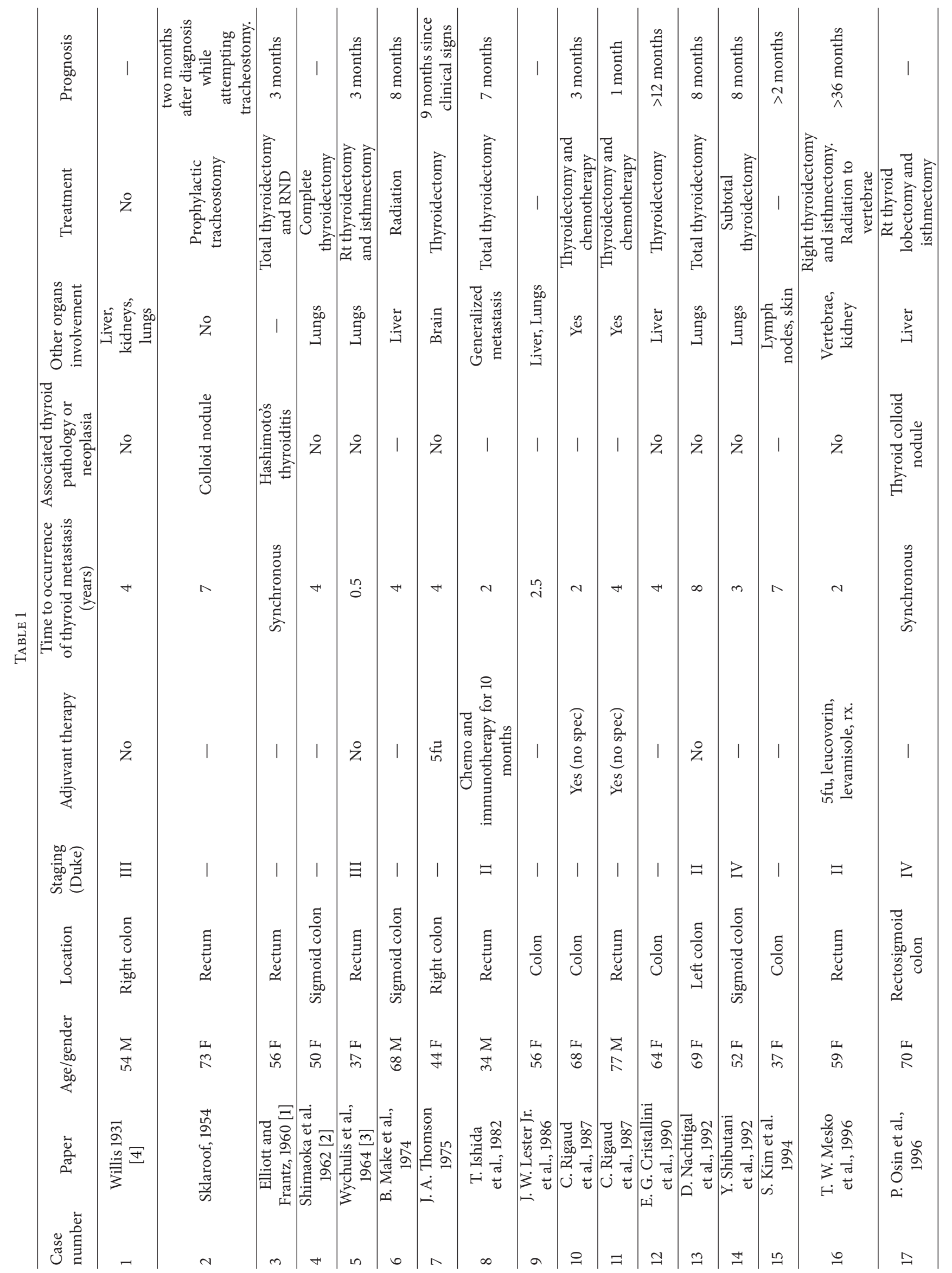




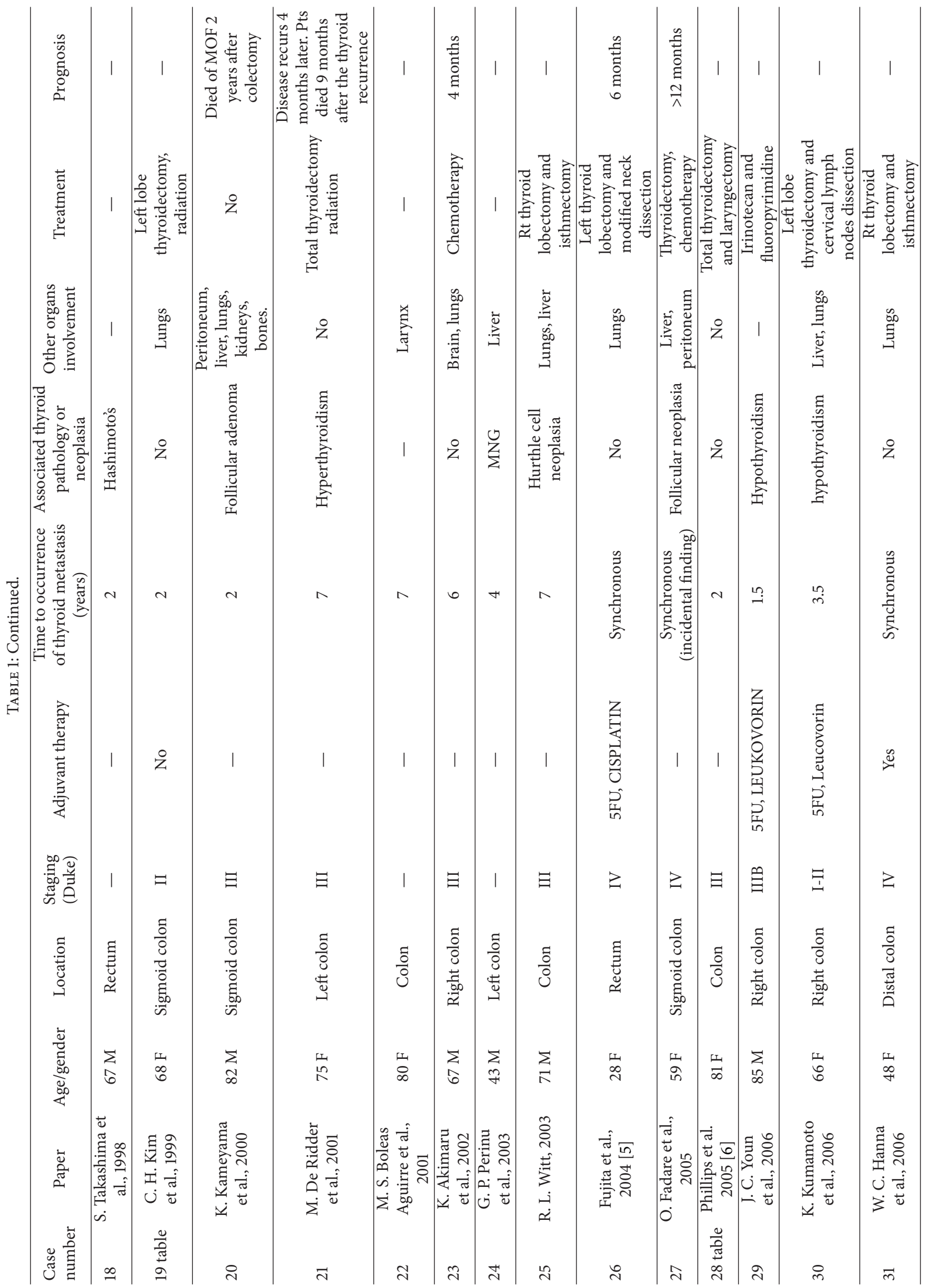




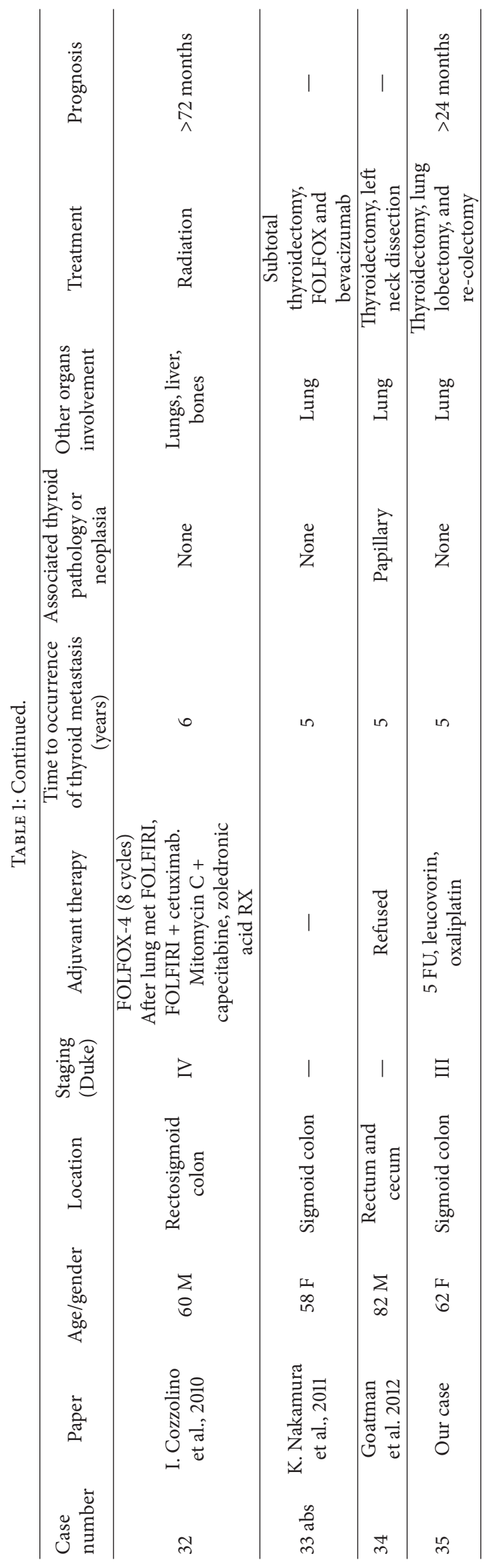


FNA revealed atypical cells without follicular or papillary differentiation. Right thyroid lobectomy was performed. The final pathological report suggested metastasis of colon carcinoma. FOLFOX adjuvant therapy was administered postoperatively.

In November 2011, an enlarged nodule was palpated on the patient's left thyroid lobe. A CT scan demonstrated a $2 \mathrm{~cm}$ nodule in the left lobe. The patient underwent complete thyroidectomy in June 2012. Final histological summation reported colonic metastasis to the left lobe of the thyroid.

\section{Discussion}

Colon adenocarcinoma metastasized to the thyroid is rare, usually detected years following colectomy, and most commonly is associated with metastasis to organs such as lungs and liver. There have been reports describing a thyroid mass as an initial presentation and thyroid metastasis without other organ involvement, but these reports are rare [5, 6]. Primary thyroid neoplasms are far more common than secondary lesions, but in the presence of other history of malignancy, the risk of metastasis is probably higher.

Cases that have been published in the literature are summarized in Table 1 . We found 34 cases, two thirds being female. Patients' age ranged from 34 to 85 . The metastasis origin was from the rectum (11 cases), sigmoid colon (9), right colon (5), and left colon (3). In 7 cases, the primary colonic location was not mentioned. The staging of the colon carcinoma was specified in only 20 reports, and at presentation there was stage III or IV in $75 \%$ of the patients. Metastasis to the thyroid was diagnosed 6 months to 8 years after colonic resection. However, in two cases, metastasis was diagnosed synchronously with the colon carcinoma. In 11 patients, a primary thyroid pathology was diagnosed in addition to the colon metastasis.

In 29 cases, other organ involvement was noted. The lung was the most frequently involved organ in cases of widespread disease, leading to a poor prognosis of survival of a few months following diagnosis. However, some reports showed a more prolonged survival of 2 or 3 years at least, as in this present case. Those patients all had diffuse disease and were either managed with chemotherapy and thyroidectomy or radiation. In our case, signs of metastasis were demonstrated by PET-CT 2 years before diagnosis but failed to be proven by FNA. This questions FNA as a reliable diagnostic modality for metastasis to the thyroid.

With regard to the primary tumor location, 19 patients had either a rectal or sigmoid adenocarcinoma. Metastasis to the thyroid could be via two routes: the portomesenteric or the caval. Some reports mentioned in Table 1 were about right sided colon carcinoma without liver involvement. This could be explained by drainage of colon metastasis to the inferior vena cava through the vertebral vessels [7].

Ten patients had another thyroid pathology, although those primary pathologies like papillary carcinoma, hypothyroidism, or goiter are not rare. On the other hand, the blood distribution to the thyroid is rich, with about 3-4\% of total cardiac output supplying the thyroid gland. The iodine-rich and hyperoxic environment in some of these primary thyroid pathologies might inhibit the development of metastatic cells by physiological and chemical mechanisms, respectively, as was suggested by Willis [4].

Although colon metastasis to the thyroid represents an advanced stage of disease and is usually associated with lung and liver metastasis, attempts should be made to reach a point where the patient can be reasonably verified with no further evidence of metastasis. Once metastasis to the thyroid is suspected by imaging, complete thyroidectomy should be performed because the likelihood of colon metastasis to thyroid in the presence of other organ involvement has been seen to be higher than that in primary thyroid neoplasia.

\section{Disclosure}

The authors and the co-authors have no financial relation with any commercial identity if mentioned in the paper.

\section{References}

[1] R. H. Elliott Jr. and V. K. Frantz, "Metastatic carcinoma masquerading as primary thyroid cancer: a report of authors' 14 cases," Annals of Surgery, vol. 151, pp. 551-561, 1960.

[2] K. Shimaoka, J. E. Sokal, and J. W. Pickren, "Metastatic neoplasms in the thyroid gland. Pathological and clinical findings," Cancer, vol. 15, pp. 557-565, 1962.

[3] A. R. Wychulis, O. H. Beahrs, and L. B. Woolner, "Metastasis of carcinoma to the thyroid gland," Annals of Surgery, vol. 160, pp. 169-177, 1964.

[4] R. A. Willis, "Metastatic tumours in the thyreoid gland," American Journal of Pathology, vol. 7, no. 3, pp. 187-208, 1931.

[5] T. Fujita, Y. Ogasawara, H. Doihara, and N. Shimizu, "Rectal adenocarcinoma metastatic to the thyroid gland," International Journal of Clinical Oncology, vol. 9, no. 6, pp. 515-519, 2004.

[6] J. S. Phillips, S. Lishman, and P. Jani, "Colonic carcinoma metastasis to the thyroid: a case of skip metastasis," Journal of Laryngology and Otology, vol. 119, no. 10, pp. 834-836, 2005.

[7] O. V. Batson, "The function of the vertebral veins and their role in the spread of metastases," Annals of Surgery, vol. 112, no. 1, pp. 138-149, 1940. 


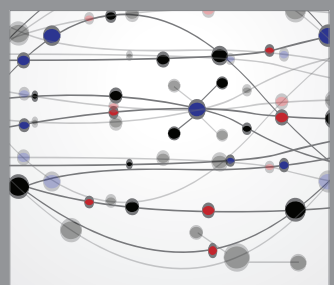

The Scientific World Journal
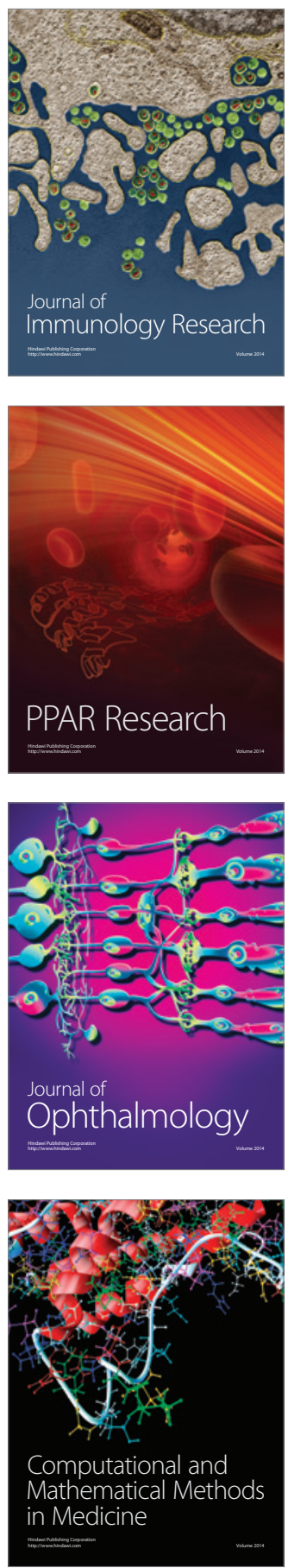

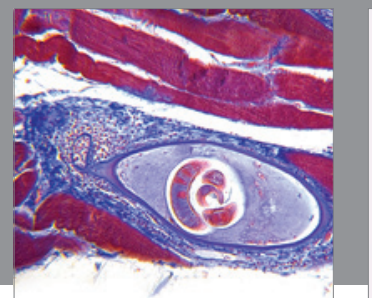

Gastroenterology

Research and Practice
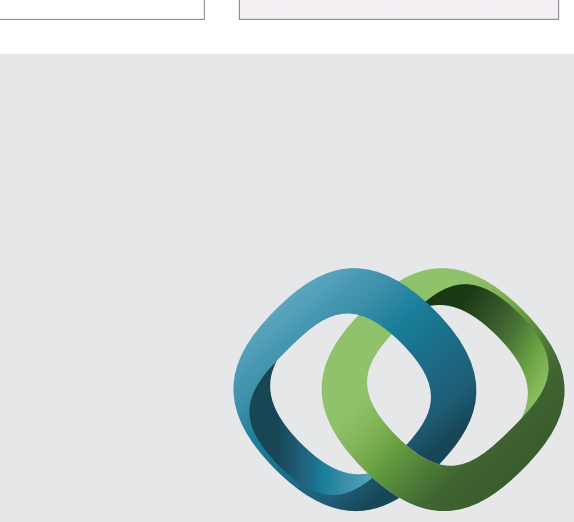

\section{Hindawi}

Submit your manuscripts at

http://www.hindawi.com
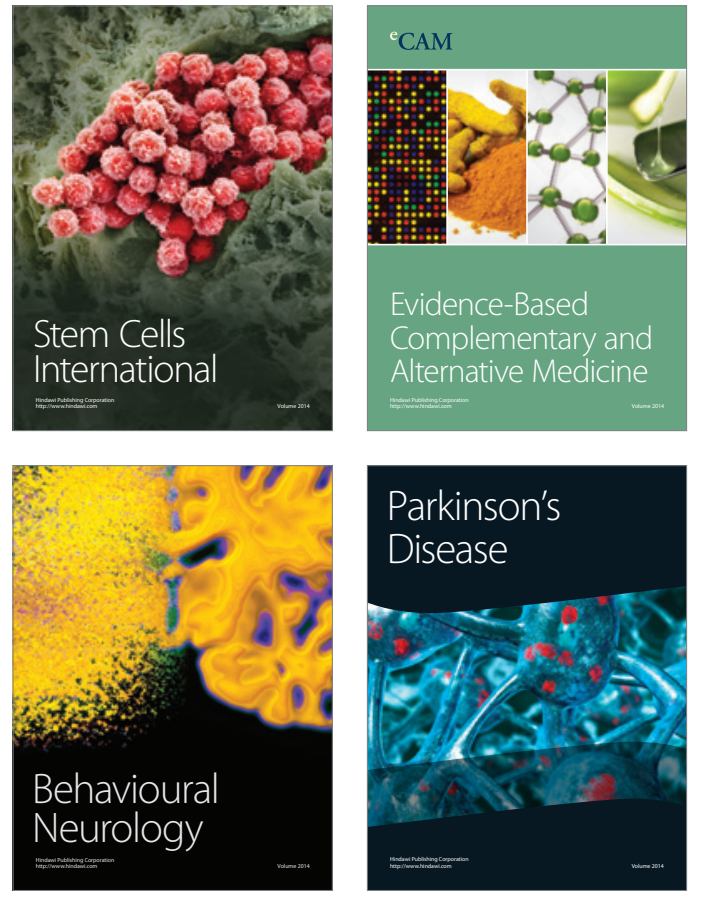
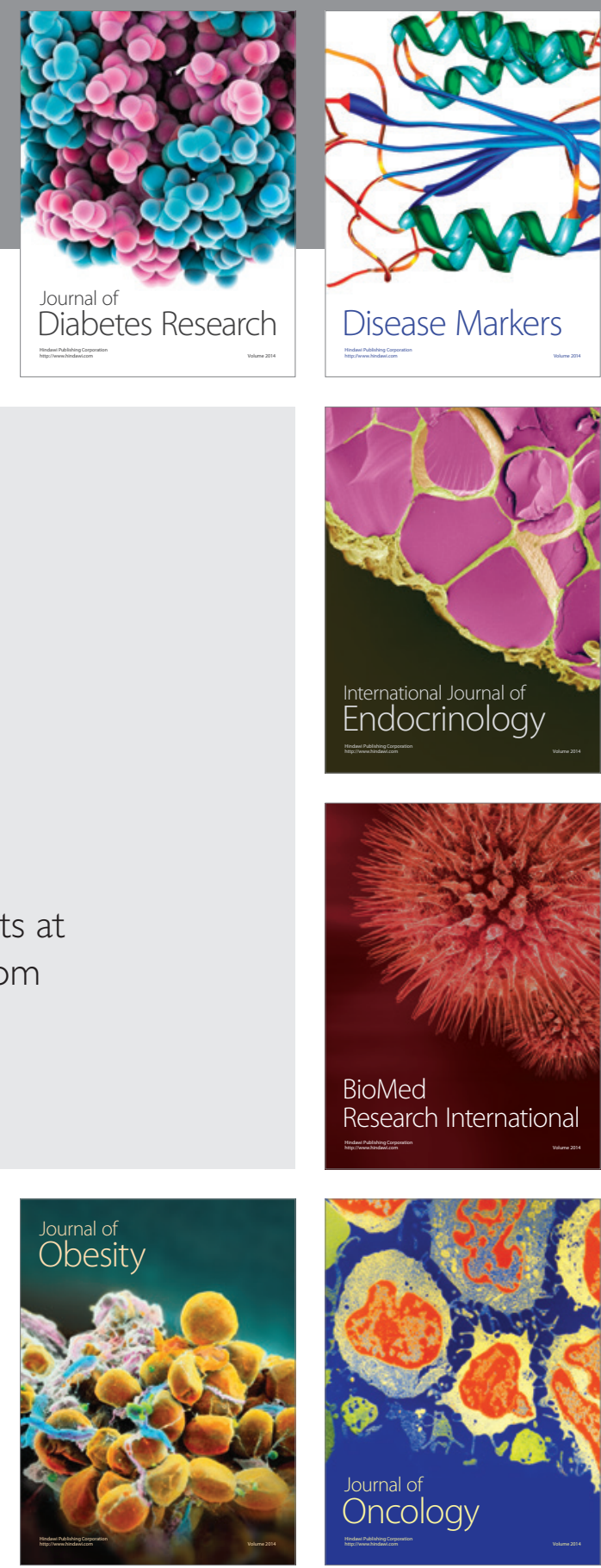

Disease Markers
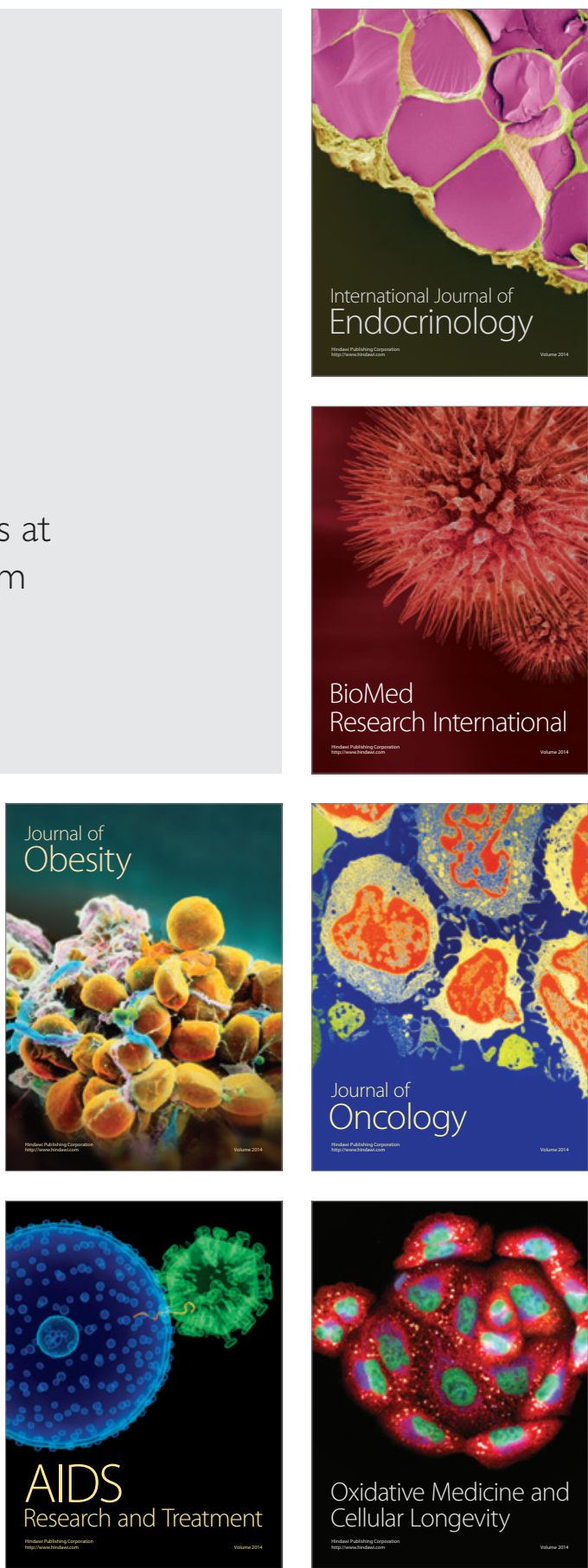